\title{
Post-secondary photonics lab: simplified portrait holography
}

\section{William Brohinsky}

William R. Brohinsky, "Post-secondary photonics lab: simplified portrait holography," Proc. SPIE 9665, Tenth International Topical Meeting on Education and Training in Optics and Photonics, 96650T (3 June 2007); doi: $10.1117 / 12.2207324$

SPIE Event: Tenth International Topical Meeting on Education and Training in Optics and Photonics, 2007, Ottawa, Ontario, Canada 


\title{
Post-secondary Photonics Lab: Simplified Portrait Holography
}

\author{
William R. Brohinsky \\ Three Rivers Community College, Norwich, CT 06360 onlyocelot@gmail.com
}

\begin{abstract}
Teaching photonics is greatly enlivened by demonstrations of practical holography. One of the more impressive varieties of display holography is the portrait hologram. However, the large number of complicated, time-consuming steps required to produce traditional portrait holograms makes it an unlikely process for demonstration and practice in a classroom laboratory environment. This paper presents a process for producing simple portrait holograms using the Denisyuk single-beam (Deep Hologram) method and a single stereoptic pair of images, which can be performed, start-to-finish, in a standard 3-hour lab period. Possibilities for expansion of the technique to larger numbers of images, potentially approaching the quality of multiple-image, master hologram-transfer hologram traditional portrait holography, as well as strategies for multiple and single beam illumination are discussed.
\end{abstract}

\section{Introduction}

A post-secondary degree program in Photonics will contain courses dealing with optics and lasers. A natural approach which combines these subjects is found in holography, usually implemented in a one-period laboratory. At TRCC (Three Rivers Community College, Norwich, CT), holography is introduced through two hands-on experiments in which a single beam Denisyuk hologram and a transmission hologram are produced. This approach is not limited to colleges, since the major producers of holography kits provide fixtures and/or directions to produce Denisyuk holograms as a first step. The addition of laser pointers to the battery of available coherent sources has decreased the cost of entry, to the point where many students entering college may have already completed a number of successful Denisyuk holograms and may be ready for something more advanced. ${ }^{1,2,3}$

One of the more impressive varieties of holography is the portrait hologram or holographic stereogram. In the canonical Benton approach, a series of black-and-white images are shot using standard photographic techniques, but in a sequence of positions along a line tangent to a circle whose radius is the nearest approach to the subject. The film is processed directly to transparencies and multiplexed into a hologram in stripes using one of various transmission hologram processes, so that each successive image in the sequence is encoded into a subsequent stripe. Care must be taken in photography to avoid keystoning, and, when striping the transmission hologram, to ensure that the stripes do not overlap and that spaces do not form between them. Mechanisms to ensure this have been developed and reported in the literature which range from simple to expensive, automated complexity ${ }^{5}$. Once processed, this hologram is used as a master in a transfer to a reflection hologram. This last step distributes each point in the master hologram across every point in the reflection hologram, eliminating the appearance of stripes. Because of the angle of view which is associated with each stripe, the resulting reflection hologram delivers a stereoptic pair of images to each eye, appropriately registered and located to give a sense of three-dimensionality. These image pairs are essentially identical to paired images used in the 19th century stereoscope (still extent today in the commercial "View Master"). However, when a sufficiently large number of views (varying in the literature from five or six to hundreds), each giving a view from a slightly different angle, are provided, the viewer gets a sense of occlusion, which is normally missing from stereoscopes.

Unfortunately, the optical setups required for making portrait stereograms and the time required for the three recording and three processing steps generally precludes their use in post-secondary class labs. In addition, the set-up for striping successive images into the master hologram requires accuracy and repeatability, in order to line the edges of each strip up exactly without overlapping or leaking light between the edges of the stripes.

Tenth International Topical Meeting on Education and Training in Optics and Photonics, edited by Marc Nantel, Proc. of SPIE Vol. 9665, 96650T · (c) 2007 SPIE, OSA, IEEE, ICO doi: $10.1117 / 12.2207324$ 
This paper describes a process for creating a variety of single-beam hologram which makes use of the angular selectivity of single-beam (Denisyuk) volume holograms to register a pair of two- dimensional images forming a stereoptic pair, overlapping, in the emulsion. The result admits reconstruction of both images using two sources of reconstruction illumination, with each of the images forming on one side of the normal to the emulsion center. Additionally, suggestions are given towards establishing a single-period laboratory exercise in which the principles of three-dimensional perception, volume holograms, and angular selectivity are introduced. Finally, some discussions of the shortcomings of current understanding of volume holograms with regards to successful geometries is given, and a wish list of future topics of exploration which might grow from the subject are introduced.

\section{The Denisyuk Hologram}

An in-depth description of the Denisyuk hologram (as a reflection, deep or volume hologram) is beyond the scope of this paper, as it is already very well covered in the literature. ${ }^{5,6,7,8}$ A simplistic overview will be provided which highlights the characteristics of volume holograms (of which type the Denisyuk is most elegant), emphasizing angular selectivity.

The single-beam volume hologram is a simple affair, illustrated in Figure 1. In recording (1a), a glass plate or a piece of film, constrained to avoid motion during exposure, is placed directly in front of an object and a suitably spatially-filtered, spread laser beam is shined through the emulsion to illuminate the object beyond. The illumination beam forms the reference beam as it passes through the emulsion and the object beam as it returns (Figure 1a). This creates standing waves in the emulsion, angled and spaced according to the angle formed between the reference and object beams. The bisector of this angle (AB) is, according to Gabor and Strokes, the angle bisector between two face-edges of a cone of greatest reconstruction, one of which face-edges forms along the axis of the reference beam. This infers that a certain amount of control is possible over the location of brightest area of reconstruction if the conditions of recording and reconstruction are controlled.

Unfortunately, simple calculations to predict the location of these areas of visibility from the geometries used in recording and reconstruction are not to be found, and this is not surprising, since most of the equations used to explain the subject are extremely complicated and are based on varying or difficult-to-discern parameters (such as emulsion thickness and wavelength). In addition, for an object of more complexity than a single point or short line segment, equations quickly climb into advanced calculus. Perhaps the best approach is to follow Gabor and Strokes' conclusions: the greatest brightness of reconstruction can be expected in a cone as described above, with two axially-concentric cones (Fig1b, e), which enclose the cone of greatest brightness $(\mathbf{C})$ and define the edges of extinction. If the problem is reduced, for instance, to the plane perpendicular to the plate's horizontal centerline and intersecting the viewer's eyes, it should be possible by experiment to find a geometry for recording an image which, when reconstructed by a single white-light illumination source, produces one image that is visible on the opposite side of plate normal(N). Using two such images, recorded and illuminated from opposing sides, will produce one image visible to each eye without the other eye's view being polluted by the wrong image. Further constraint, namely, reducing the recorded images $(\mathbf{O})$ to 2-dimensional images printed on paper, is expected to reduce experimental complexity further, although in fact this may further complicate the math.

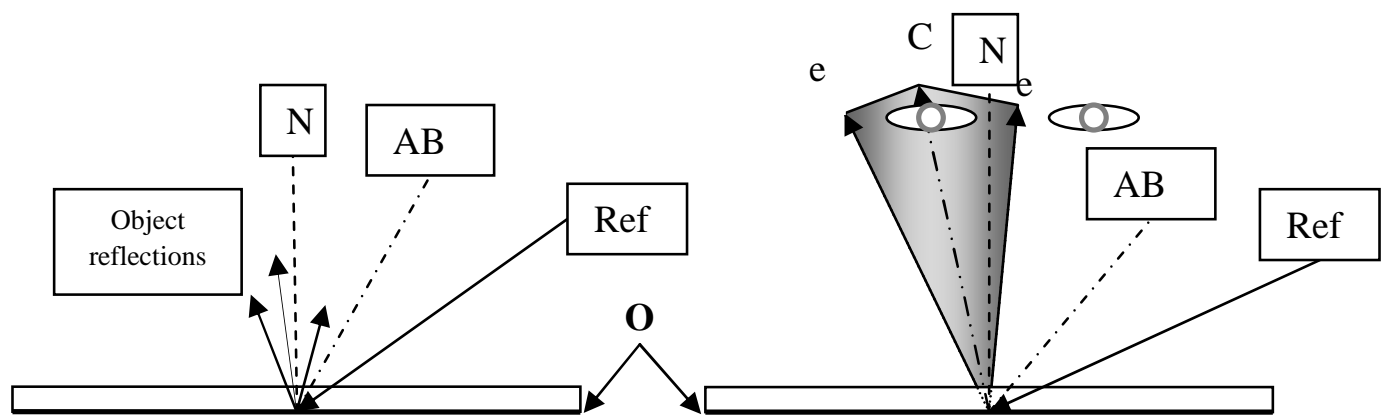

Figure 1. Schematic showing Image $\mathbf{O}$ pressed against the emulsion side of the plate, illustrating recording and reconstruction of one image.

Experimentation proved to be the only way to determine a working geometry. 


\section{Experiments to Establish a Geometry for the Denisyuk Stereogram}

The first step was to prove that a plane image pressed against the emulsion of a glass plate (Slavitch PFG-03M) could produce a reconstructable image, viewable in white-light. Further, these tests looked for a clear extinction point where the image would cease to be viewed, based on angle to plate-center normal in the $\mathrm{x}$ axis. The first image was made of a slide rule, because the object was of high contrast, had fine markings, and was solid. The results were very satisfactory.

Subsequent tests used images printed on various grades of printer paper by a laser printer. The early results were disappointing, until the "bad spots" were recognized as interferometry. Using a second glass plate to back the image paper and butterfly clips to hold the plates together helped to stabilize the paper with the image. Additionally, long (10 minute) rest periods after placing the plate on the table allowed the plates to equalize in temperature eliminating movement caused by the minute changes in size of the glass. This eliminated most signs of interference.

Finally, an experiment using paper images which indicated the angle of recording illumination to the table (later translated to angle-to-normal) was made, processed, and tested with illumination at various angles and from various viewing angles.

The plate marked for an angle of $+60^{\circ}$ to plate normal (i.e., with the illumination source to the right of the plate and at the same $60^{\circ}$ angle that was used for recording) was found to produce a left-side-of-normal image which abruptly disappeared about $13^{\circ}$ to the right of the normal when a single-point viewer (one eye) was brought across the plate from the left. This plate was rotated around the normal by $180^{\circ}$, and when illuminated with the same reconstruction beam angle, but also rotated around normal by $180^{\circ}$, produced an image to the right of center, which also extinguished for a single-point of observation moved from right to left. When viewed from the other side of plate normal, in both cases, the image did not reconstruct. In reconstruction, the reconstruction area (between the extinction cones) can be shifted away from the angle bisector by increasing the angle-tonormal of the reconstruction beam. Placing the illumination source at approximately $70^{\circ}$ to plate normal shifted the extinction point to within $2-3^{\circ}$ of plate normal.

This indicated that, for conditions in the author's home lab, using Slavitch PFG-03M plates and JD4 processing chemicals, a proper Denisyuk stereogram could be recorded using $60^{\circ}$ as the illumination angle to normal, illuminating from the right side to record the left image and the left side to record the right image, and the result, when viewed straight down the plate centerline normal and illuminated from near to $70^{\circ}$ from both sides should produce a viewable stereoptic pair with minimal crosstalk between the two images.

\section{Using Denisyuk Portrait Stereograms for a Post-Secondary Laboratory Experiment}

The exact geometry to be used must be determined beforehand, since the angle of illumination given in this paper was specific to the laser, emulsion and chemistry used in the author's lab. This might be an excellent extra-credit lab for an advanced student or student group.

For a completely new installation using different emulsions (or film instead of plates) or different chemistry, a recommended approach is to record a set of Denisyuk holograms with the reference beam at varying angles of illumination from normal. The angles could be established by a mirror on a pivoting, lockable extension, which can be rotated around the hologram to redirect light from a fixed laser to the plate. In such a case, a horizontal slit placed just after the spatial filter can be used to make a line of light on a piece of vertical cardstock aligned along the $\mathrm{x}$ axis of the plate-position midpoint, which can be marked beforehand with the angles intended. Likewise, with a laser pointer, it is feasible to mount the pointer on the pivoting arm itself. In this case, the laser can be fixed to the arm and the arm's angle-to-normal (with a slight adjustment for elevation of the pivot point) 
can be used directly. The author is blessed with a laser diode pigtailed to single-mode fiber at the diode's wavelength $(658.6 \mathrm{~nm})$, and has mounted the fiber connector to his pivoting rod, using a small, clean, negative lens to increase the spread of the beam over the NA of the fiber. With only the weight of the connector and fiber on the rod itself, very little is required to lock illumination in place.

Once a desirable angle is determined, a Denisyuk stereogram can be made using a geometric stereoptic pair (such as a wire-frame cube) to record right and left images. This can be used to determine if the resulting right and left images have sufficient brightness at the position of the viewer's eyes, and ensure that image overlap is acceptable. It is not necessary that the image extinction edges are precisely aligned, it is sufficient that balanced views of each image are presented to the appropriate eye without crosstalk.

The following is an example of a possible lab scenario. It should be mentioned that, even with the simplifications, this lab can be lengthy. It is desirable to distribute the lab sheets before the lab starts and encourage the students to have familiarized themselves with the particulars before beginning the lab period. However, since many of the steps can be performed in parallel, or en masse (such as development and bleaching), it is quite possible for each member of a lab group to make their own Denisyuk Stereogram within the given lab period. The laboratory manual produced by Project PHOTON2 (NSF/ATE \#0302528) included a procedure to produce a Denisyuk hologram using materials included in the PHOTON2 lab kit. The procedure that follows is an adaptation of the PHOTON2 experiment, which assumes students have already completed the basic Denisyuk hologram experiment.

$<<$ Beginning of example lab $>>$

\section{Denisyuk ("single-beam") Portrait Stereogram}

\section{Safety Notes}

Do not look directly into the laser cavity, or at any reflections of the laser caused by shiny surfaces. In this experiment, the beam will be aimed at the optical table: so spread the beam first, then angle it towards the table, to avoid powerful specular reflections! Limit personnel in the lab to the minimum number required to perform the lab.

\section{Preliminary notes}

Have all developing chemicals mixed and arranged in the order that they will be used before beginning. Be sure to follow directions on the packages. NOTE: Bleach, rinse water and photo-flo solution do not degrade, so they can be mixed well beforehand and left standing in covered trays. Part A and B can be made beforehand if they are refrigerated, but should be mixed when the lab begins.

Check alignment of the optical system before exposure - then check it again.

The safelight for red sensitive holography film is green. A green nightlight, placed away from the film developing process, will work well.

During exposure, the entire optical set-up must remain vibration free. Ensure the set-up has had plenty of time to settle and equalize to room temperature from handling. Do not move around, make noise, or touch the table during exposure.

Be sure to dispose of spent chemicals safely, following all state and local regulations. 


\section{Objective}

- $\quad$ To create and view a Denisyuk (single-beam) holographic stereogram.

\section{Equipment and supplies}

630-660nm (or a HeNe laser beam which is spread by a spatial filter, negative lens or reflection from a concave mirror)

Laser mount or connector adapter with appropriate posts, swivels and post holders

Digital camera

Computer with digital camera interface (or card reader) and appropriate software (the Gimp or Photoshop)

Rare-earth magnets to secure the edges of the plate during recording (voice-coil magnets from an old hard disk drive are ideal! Cover them with rubber tape to reduce the stress on the plate edges.)

Opaque cardboard for shutter and light-stops

Matte black spray-paint

Two illumination sources, White light point sources with adjustable supports

\section{Theoretical background}

Photography captures a 2-dimensional image of a scene, focused through a lens and aperture to place the image plane on the film or sensor. Grey-scale pictures (black and white) with acceptable representation of skin tones are easily produced by weighting the film or sensor's response to different colors or by computer post-processing. If two images of a scene are captured by photography from slightly-different locations (similar to the position of a human's eyes) the resulting pictures can be superimposed by presenting the appropriate picture to each eye. These images are called a stereoptic pair. The mind of the viewer will knit the two images into one, perceiving three-dimensionality from slight offsets in recognizably identical objects between the two pictures. This three-dimensional presentation lacks two important features of depth-vision, occlusion (further things disappearing behind nearer things as you move your head) and accommodation (focus-changing which provides another clue to depth), and yet it has been a popular method of providing 3D views for over a century. (The primary steroptic viewer, the traditional Stereoscope was designed by author Oliver Wendell Holmes in the late $19^{\text {th }}$ century!)

Holography captures the entire scene in the emulsion, complete with dimensionality, by interference between the reflected object light and the mutually-coherent reference beam. However, the monochromatic characteristic of laser light (which cannot capture skin tones), coupled with its capacity for large amounts of energy, make it ill-suited for taking holograms of living subjects. Further, the small movements of air currents or relaxing textiles make many objects unsuitable for hologram subjects.

The solution to the problems of direct-laser-illumination of portrait subjects has been solved by holographic stereograms. This process uses multiple pictures of the subject, each taken from a slightly different position, and encodes them into a hologram in stripes. Each stripe acts like a vertical window into the subject space, presenting a slightly different perspective. Taken together, these images provide a sense of smoothness (like the still frames of a movie, when viewed above the "flicker rate"), allowing a sense of perspective, parallax, occlusion and, because each stripe reconstructs the object wave, requiring the eye to focus differently to resolve objects at different virtual depths, accommodation. The process is also lengthy, requiring special photographic processing, complex optical setups, and recording of two holograms. 
The Denisyuk Stereogram which you will produce in today's lab is a simplification of this process, but retains the significant features of a holographic stereogram: two images, taken with a digital camera, will be encoded into a Denisyuk hologram so that each image reconstructs for one eye of the observer.

In the Single-Beam Reflection Hologram lab, you have already created a Denisyuk (singlebeam) hologram. This type of hologram is viewable in white light, but you may have noticed that there are angles of illumination which do not recreate the hologram image. Likewise, you may have noticed that there are angles of viewing, from which the image is not visible, and that the change from bright reconstruction to no reconstruction at all is quite abrupt. This is the result of angular selectivity, which is a characteristic of reflection holograms. By changing the recording illumination angle, multiple images can be stored in one hologram and selectively reconstructed by changing the angle of the reconstruction reference.

Angular selectivity in reflection holograms comes from the similarity of the fringes which form in the emulsion to volume gratings. In general, the angle formed between the object beam (extended through the emulsion) and the reference beam establishes an angle bisector. When the image is reconstructed, the brightest image will form an angle with the illumination reference beam which has the same bisector. Additionally, the image of reconstruction is brightest here, but falls off on either side.

If the illumination for recording is angled approximately $60^{\circ}$ from plate normal, for an object which comprises a two-dimensional image pressed against the emulsion of a PG-03M holographic plate (Slavitch), the resulting image (with a white-light illumination source near $80^{\circ}$ to normal) will reconstruct near the opposite side of the plate normal. This is shown in figure L-1.

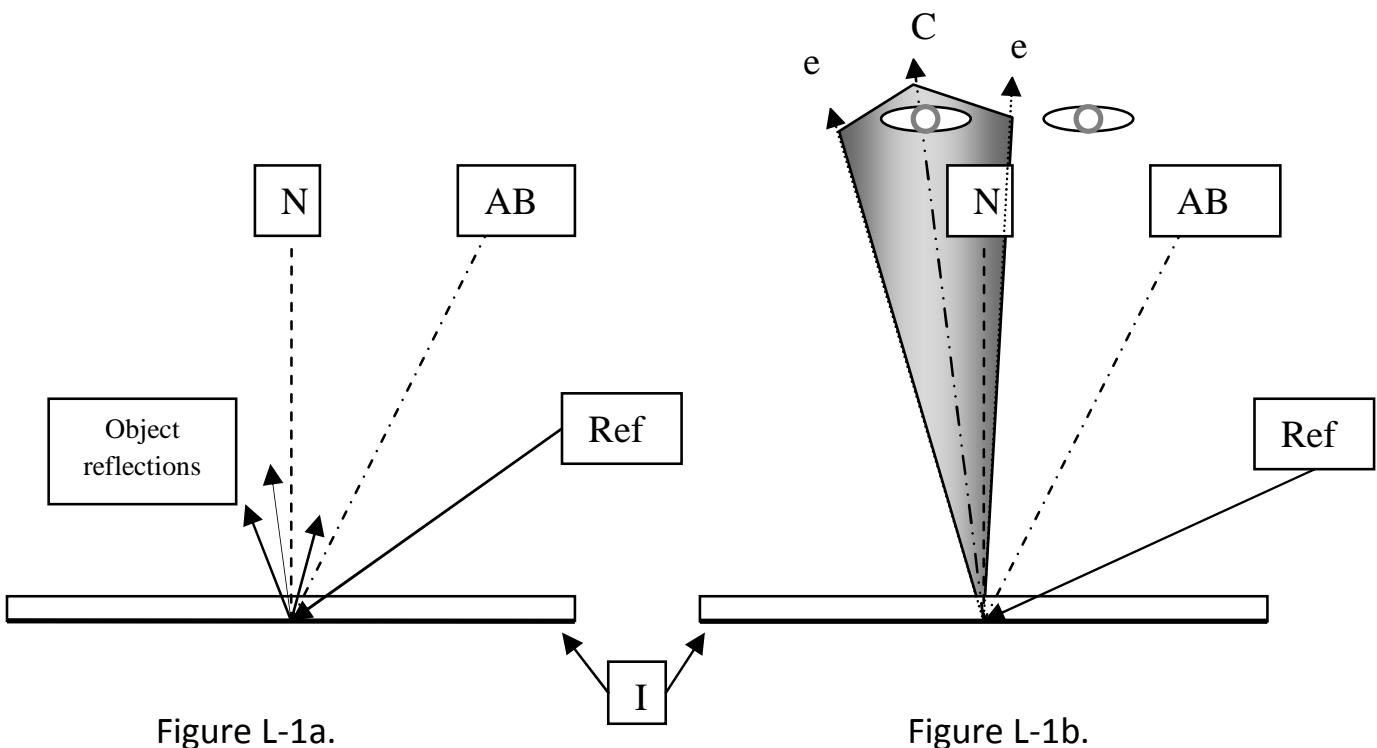

Figure L-1. In recording, the reference illumination Ref passes through the plate and emulsion, strikes the image $\mathbf{I}$ and returns almost immediately. Because of the close proximity of the image to the emulsion, the predominant reflection acts near-to-parallel to the normal $\mathbf{N}$. The resulting interference pattern, a complex volume grating, develops an encoded angle bisector (roughly $60^{\circ}$ in this case). When the hologram is illuminated (at a slightly different reference angle) by white light, the reconstruction forms in a cone around the angle bisector (only the left cross-section of which is shown, for clarity). The bounding curve of this cone C identifies points of maximum reconstruction brightness, with two concentric cones e marking the boundaries on either side where extinction takes place. By increasing the angle of the reconstruction illumination, the cone of maximum brightness is skewed in the opposite direction from normal, to the left side. The left eye in Fig. L-1b sees the image, while the right eye does not. 
The two images which will each be encoded into the single holographic plate will be recorded with reference illumination from opposing sides of the plate normal, so that their reconstruction cones will form on either side of the plate normal. Since the reconstruction forms to the left for right-side recording illumination, and to the right for left-side illumination when illuminated from one side with white light, we will be using the geometry shown in figure L-2. For simplicity, inverting the second image and rotating the plate $180^{\circ}$ about its Normal will allow using a fixed recording reference beam.

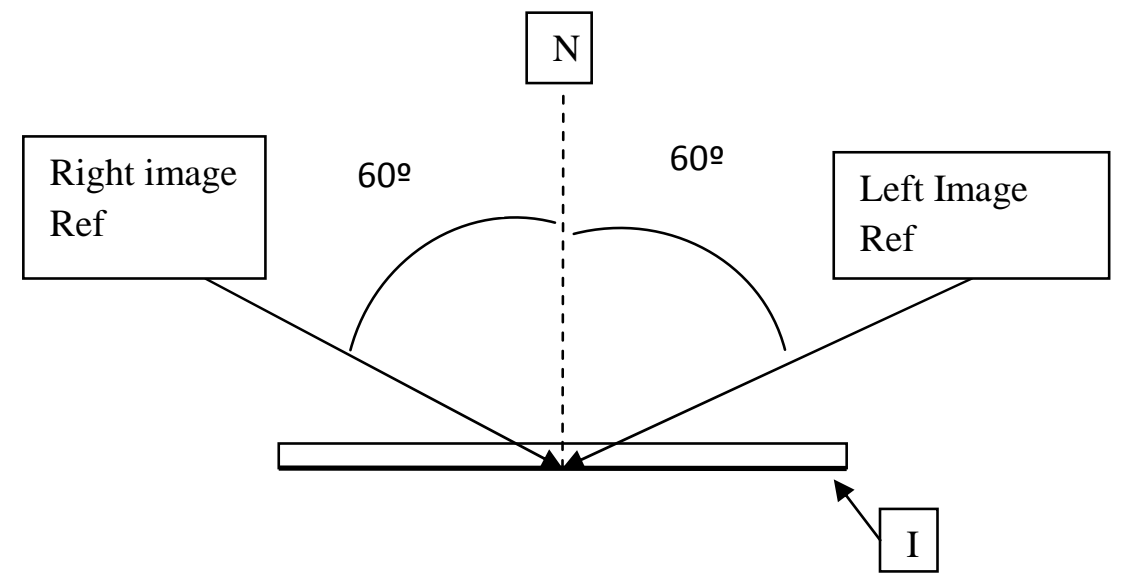

Figure L-2. Each image is recorded with a reference beam coming from the opposite side of plate normal. In practice, rotating the plate and second image allows using a fixed reference illumination. Reference rays represent spread, filtered beams.

This geometry will yield a hologram in which two images are recorded. The two images will be reconstructed with a pair of illumination beams from opposite sides at whatever angle provides the best image visibility for each of the viewer's eyes, as in figure L-3.

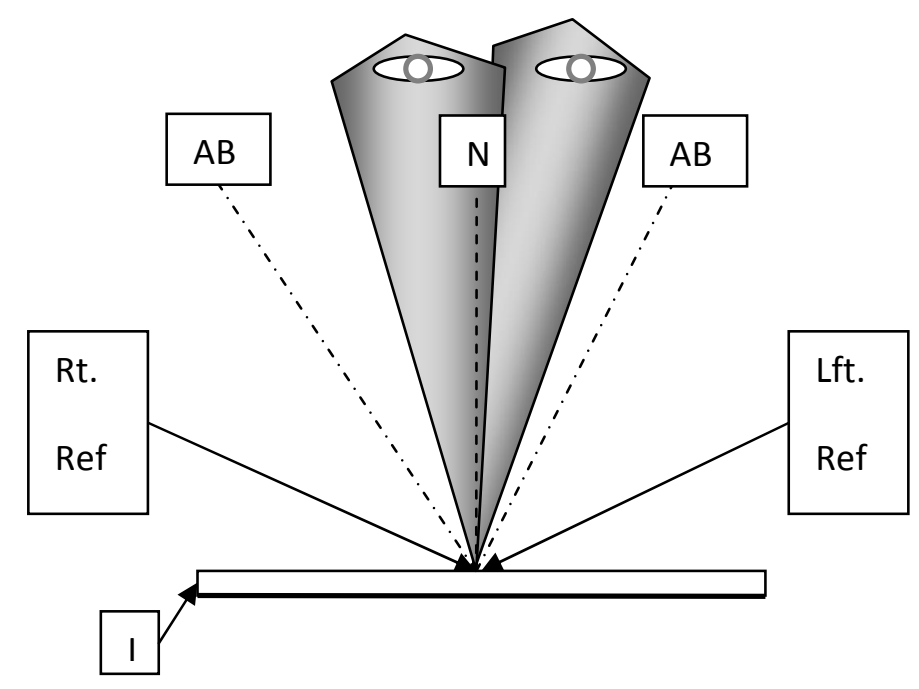

Figure L-3. On reconstruction, the visibility spaces formed will superimpose the proper image on each of the viewer's eyes, the right image for the right eye, and the left image for the left eye. Angling the reconstruction illumination beams further from plate normal deflects the reconstruction away from the angle bisector $\mathrm{AB}$, shifting the extinction point near to the plate normal, and making the left image reconstruct for the left eye but not the right eye, and vice versa. 


\section{Procedure}

NOTE: This procedure assumes the use of PFG-03M film plates (Slavitch) and JD-4 developer, available from Integraf (http://holokits.com/). If you use different film or developer, consult the manufacturer's instructions.

\section{Set-up}

1. Pour enough developer (equal parts $\mathrm{A}$ and $\mathrm{B}$ ) into one developing tray to cover a film plate (around $2 \mathrm{~cm}$ deep.) Pour a similar amount of bleach into a second tray. Fill a third tray with water only and add a few drops of Photo-flo. Have a source of running water nearby. (If no running water is available, a large bucket of clean tap water will do.)

2. Arrange the counter so the film plates and developing trays are readily accessible. Clear away any other objects. Remember, you will be developing film in the dark!

3. Set up the laser, any required lenses, mirrors, holders, etc. to provide illumination of a spot on the table slightly larger than the plates. The incoming angle of this spread beam should be $60^{\circ}$ to the table normal (i.e., $30^{\circ}$ from the table surface). (Placing the illumination $8 "$ above the table and directing it down at a point 14 " away from the deflection point will provide an angle of $30^{\circ}$ to the table.)

4. Choose a suitable subject: lab partners make good subjects! Take two pictures of them with the digital camera using the "shoot-lean-shoot" procedure. Ensure the camera is level and moves horizontally between the two images by about 4".

5. Transfer the images to the computer, convert them to grey scale and size them to print out at the dimensions of the plate. Place the images side-by-side for printing and test them by looking past the images (through the screen) until the images superimpose. (If this is difficult, it is possible to arrange the images so the right-eye image is on the left: then cross your eyes to overlap the images.) When the images are superimposed and your eyes grow accustom to them, they should fall into focus and you will see the three-dimensionality.

6. Print the images and cut them out. Trim them to exactly the dimensions of the plates being used. Rotate the right-eye image $180^{\circ}$ and tape it to the table so that it is centered in the spot. The right side of the image should be toward the diversion point (or laser if direct illumination is used) and perpendicular to a line between the center of the spot and the deflection point. Tape all four sides so that the paper lies flat. Then, tape the other image over the first (the top of the second image should be in the same direction as the bottom of the first) and tape only one side to the table.

7. Block the beam with the shutter, turn off all lights but the safelight, and close doors and light barriers.

8. Take a plate from the darkbox, and close the box completely. Determine which side of the plate has the emulsion (the emulsion is sticky when touched with a moistened finger.)

9. Place the plate over the upper image so that it aligns with the image edges, with the emulsion side down. Place the magnets so that they grip the edges of the plate from above and compress the plate onto the images.

10. Wait for at least 10 minutes for the plate to equalize temperature from handling. Lift the shutter and hold it so it still blocks the beam, without touching any part of the table or optical set-up for at least 15 seconds to allow the table to settle from any vibrations.

11. Lift the shutter completely to expose the plate for about 15 seconds.

12. Replace the shutter.

13. Remove the magnets and lift the plate. Rotate the plate $180^{\circ}$, and remove the top image, tape-andall. Place the plate on the lower image and align the edges. Replace the magnets so that the image is compressed under the emulsion side of the plate.

14. Let the table settle and the plate equalize temperature for 10 minutes.

15. Lift the shutter, and as before, continue to block the beam for at least 15 seconds.

16. Lift the shutter completely out of the way and expose the plate for 15 seconds.

17. Replace the shutter. 


\section{Development:}

1. Put a plastic glove on the hand you will use for developing the plate.

2. Develop for 1 minute in the $A+B$ developer mix. Hold the plates at the edges and agitate by shaking back and forth with the emulsion completely submerged. Rotate the plate from time to time to expose the edges that were covered by your fingers.

3. Rinse for 1 minute.

4. Bleach for at least 15 seconds after the plate is clear in the safelight.

5. Rinse again, then dip in photo-flo for 5 seconds.

6. Air dry the emulsion. The holograms will not be visible until the plate is entirely dry. The impatient can employ a hair dryer, but be careful not to hold it too close.

7. When the emulsion is completely dry, spray-paint the emulsion side with black, matte paint. Although this coating is not absolutely necessary, it will vastly improve the results.

\section{Viewing:}

Use two matched point sources of light (a light bulb will work at sufficient distance) placed at about $70-75^{\circ}$ from plate normal on either side of the plate. Viewer position should be with the plate center directly in front of the viewer. Distance from the plate may be dependent on the viewer. Test the two images by closing one eye at a time. There should be an image visible for each eye, without two images appearing to one eye. Adjust the location of the light sources for the best image for each eye which doesn't produce an image for the other eye, turning on one source at a time. Once this viewing point is found, turning on both sources and opening both eyes should reveal a 3-dimensional image!

\section{Conclusion}

Describe the differences between the Denisyuk stereoscopic hologram and a "real" hologram. What is missing? Did your two images register properly and superimpose with a minimum of viewer strain? Was the color of each image identical? What might have caused a difference? Can you think of ways to improve this process?

The portrait holograms in the hall display and those which we viewed on our visit to the MIT Holography Museum are made differently from the Denisyuk stereogram. Where our Stereogram has only two images encoded into it, the traditional portrait hologram contains many views. Each of these images is "striped" into a transmission hologram, and the resulting multiplex transmission hologram is transferred to a reflection hologram to create the white-light viewable hologram you saw. What would be necessary to be able to store more images into a Denisyuk Stereogram?

$<<$ End of example laboratory >>

\section{The Future}

The Denisyuk Stereogram process currently can reliably produce a dual-image stereogram. Further work is necessary to discern a simple geometry, which can be expressed in understandable math, which will predict the width between the extinction cones, allowing control over how many images could be multiplexed into a single Denisyuk hologram. Additional simplified calculations that will predict the required reference beam angle and allowable deviation from spherical or parallel wavefronts required to produce a visible region with desired extinction edges in three dimensions are much to be desired. The more images that can be stored in a holographic stereogram, the more smooth the transitions between views, and the more 'natural' the appearance of the resulting 3D image.

Multiple-beam illumination is required for this kind of Denisyuk stereogram. A single-illumination approach is desirable. None-the-less, there are applications where multiple fixed, selectable illumination sources working on one Denisyuk stereogram with a different stereoptic pair encoded to each angle of illumination might be 
interesting. One such possibility would be super-imposed images, selected by the presence of illumination from specific angles, causing (for instance) a bottle to appear and disappear in a subject's hand. Other combinations of stereoptic pairs, singly or in combination, might be possible.

\section{Acknowledgements}

The author would like to acknowledge the help he has received from many people, including Dr. Tung Jeong and Mark Kahan, who suggested and provided papers which had significant impact on the development of the theoretical background for this project, Denis Leonard of IPG, who provided a red pointer-laser which was used as the laser illumination for all experiments, my wife Deborah Brohinsky, for proofreading and continuity (and the patience of a saint) and of course, my advisor, Judy Donnelly, without whose encouragement this process would never have been developed.

\section{References}

[1] Jeong, Tung H., "Making Holograms in Middle and High Schools," Proc. Sixth ETOP Conference, SPIE Vol. 3831 (2000), p. 233-228

[2] Hansen, Uwe J. and Swez, John A., "Holography in the High School Laboratory", SPIE Vol. 2525 (1995)

[3] "West Mesa Highschool :Technology Academies," Holography curriculum within Course "Intro to Photonics", web page http://www.aps.edu/aps/wmhs/atca/acad_photo_com.html\#holography, visited 4/14/2007

[4] Saxby, G, "Practical Holography", 3E, Institute of Physics Publishing (2004), ISBN 0750309121

[5] Molteni, W. J., "Shear Lens Photography for Holographic Stereograms", SPIE Vol. 1461 Practical Holography V(1991), p132-141

[6] Gabor, D. and Stroke, G. W., “The Theory of Deep Holograms”, Proc. Roy. Soc. A.304, p275-289 (1968)

[7] Syms, R. R. A., "Practical Volume Holography", (Oxford Engineering Science Series, 24) Oxford University Press (1990), ISBN 0-19-856191-1

[8] Hariharan, P, "Basics of Holography”, Cambridge University Press (2002), ISBN 052180741 